\title{
Spectrum Sharing and Operator Model for Rural and Remote Area Networks
}

\author{
Johanna Vartiainen, Marja Matinmikko-Blue, Heikki Karvonen \\ Centre for Wireless Communications \\ University of Oulu \\ Oulu, Finland \\ firstname.lastname@oulu.fi
}

\author{
Luciano Mendes \\ Radiocommunications Research Center \\ Inatel \\ Santa Rita do Sapucai, Brazil \\ luciano@inatel.br
}

\begin{abstract}
Connectivity in remote and rural areas is a challenge which is not sufficiently tackled by the on-going development of $5 \mathrm{G}$ networks. Alternative operator models that promote the establishment of locally deployed networks to serve the underserved are important to complement the mobile network operator (MNO) driven connectivity market to serve the underserved. This paper describes the connectivity challenges that remote and rural areas face and proposes a generic spectrum sharing model that allows local deployment of $5 \mathrm{G}$ networks with new local operator models. The proposed approach consists of different levels of spectrum access rights that are realized with on combined use of a geolocation database to protect the incumbent spectrum users and spectrum sensing to facilitate sharing between the local $5 \mathrm{G}$ networks. The propose approach allows different stakeholders to become local rural and remote area operators by gaining access to the spectrum locally through shared access to the spectrum.

Index Terms - spectrum sensing, database, remote area.
\end{abstract}

\section{INTRODUCTION}

Affordable connectivity in rural and remote areas is a global challenge. The 5G network deployments reported by the mobile network operators (MNOs) are currently targeting high-demand areas with little emphasis on how to connect the unconnected. Alternative operator models to complement the MNOs are emerging to allow connectivity in challenge areas [1]. The emergence of rural and remote area networks is highly dependent on the availability of spectrum, which in the case of mobile communication networks is centered into the hands of a small number of MNOs. On the other hand, those areas typically do not have abundant wireless usage, which allows the establishment of new locally deployed networks by making spectrum available through shared access to the spectrum.

There are many techniques developed for spectrum sharing including spectrum sensing, cooperative spectrum sensing, geolocation databases, and the use of beacons [2]. These techniques can be used under different regulatory models that define the rules and conditions for shared access to the spectrum. The use of TV white spaces (TVWS) has attracted interest in industry, regulation and research domains as the

This research has received funding from the European Union Horizon 2020 Programme (H2020/2017-2019) under grant agreement N0. 777137 and from the Ministry of Science, Technology under the 4th EU-BR Coordinated Call Information and Communication Technologies through 5G-RANGE project. In addition, this research has been financially supported in part by Academy of Finland 6Genesis Flagship (grant 318927). means for attaining wide area coverage due to operations in lower frequency bands but the MNOs preferred gaining exclusive rights and it did not become a wide-spread model for the deployment of cellular networks.

While there is a considerable amount of research on the use of TVWSs with geolocation databases and more recently databases combined with sensing [3], [4], there is very little research on its application to remote and rural area scenarios especially in the context of 5G. Since these lower frequency bands are particularly suitable for remote and rural areas, this paper looks into how these bands could be made available for local networks. We introduces an alternative operator model for remote and rural areas and propose a spectrum sharing model that is based on the combined use of databases and sensing that would allow different stakeholders to become local $5 \mathrm{G}$ operators.

The rest of the paper is organized as follows. Section II introduces the considered remote and rural area scenario. Section III summarizes recent spectrum sharing models. Section IV proposes an operator and spectrum sharing model for the remote and rural area scenario and Section $\mathrm{V}$ goes into the details of the role of database and sensing in the proposed spectrum access model. Finally, Section VI concludes the paper.

\section{Remote And Rural Area Scenario}

Today, there is a big connectivity gap between urban and rural areas. People living in undeserved or uncovered areas are segregated from the digital era and they cannot profit from the benefits that a reliable Internet access can offer. In continental size countries, such as Brazil, this lack of coverage in remote and rural areas creates severe social and economic problems. People living far away from cities and towns are likely to remain aside from the digital world, without access to new opportunities and unable to contribute with the digital society. Also, a reliable rural area coverage is essential for the smart farming development. The food demand is growing all over the world. In Brazil, which is one of the biggest food producer in the world, the growth in production leads to an increase in the area used for agribusiness. This process causes a severe pressure on the environment and compromises important ecosystem, such as the Amazon Forest. The solution 
for this dilemma is to increase the productivity of the area already used for agribusiness. In order to achieve this goal, it is important to collect data from the fields, such as soil humidity and $\mathrm{pH}$, local rainfall levels, and wind strength and direction. Also, information about the machinery used for seeding, harvest, and agrochemicals spraying must be provided in real-time to guarantee high efficiency of the processes. Finally, drones can provide real-time footage from the fields and cloud-based algorithms can be used to identify areas under lack of water or under attack of plagues. All these actions can be used to increase the agribusiness productivity in a system called smart farms.

One of the main problems in providing a reliable and costeffective mobile network in remote areas is the high price of the licensed spectrum which restricts who can operate a cellular network. One approach to overcome this issue consists on exploiting the TVWS in an opportunistic way. In Brazil, Anatel (National Telecommunications Agency) is responsible for the authorizing and inspect the spectrum usage. Recently, this agency has announced that the TVWS usage in Brazil will be discussed with the broadcasters, academia, telecommunications operators and industry. The idea is to define a TVWS exploitation model, which can be regulated by 2021 . This is an important milestone in the process to close the Internet connectivity gap and improve the agribusiness efficiency in Latin America, since Brazil is a regional reference.

A generic high-level communication scenario for remote and rural area use cases is illustrated in Figure 1. There are various use cases for rural and remote areas in 5th generation access networks as introduced in [5]. Assume that in Figure 1, use case 1 includes a base station (here denoted as 5GRANGE BS) connected to wireless backhaul, and there are several UEs (blue boxes) in the covered area. Use case 2 can be considered as a remote healthcare, or voice and data connectivity scenario at rural village where there are also programme-making and special events (PMSE) signals (like wireless microphone) signals present, which affect some UEs (red boxes) in that cell. Use case 3 can be considered as a smart farm deployed using long-range technology. As Figure 1 illustrates, all cells are affected by DTV signals, which should not be interfered by SU. In addition pirated DTV signal is affecting two cells. Spectrum holes detection and interference avoidance must be enabled by combined spectrum sensing and database approach.

\section{Existing Spectrum Sharing Models}

Several spectrum sharing models exist for PUs (IU) and SUs with different levels of access rights. A summary of the main spectrum sharing concepts is presented in Figure 2, where three different models with the different levels of access rights are illustrated. US Citizens Broadband Radio Service (CBRS) is a dynamic spectrum sharing model defined by Federal Communications Commission (FCC) [6]. CBRS access model consists of three tiers with different levels of spectrum access rights: Tier 1 includes IUs, Tier 2 includes priority access that operates in a specific geographical area, and Tier 3

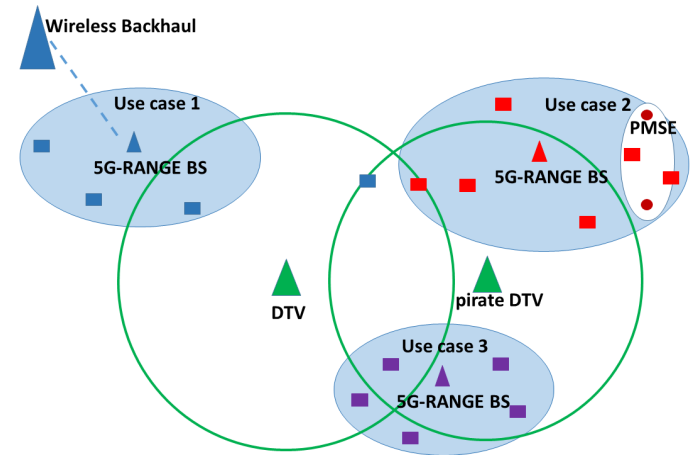

Fig. 1. Generic spectrum sensing scenario.

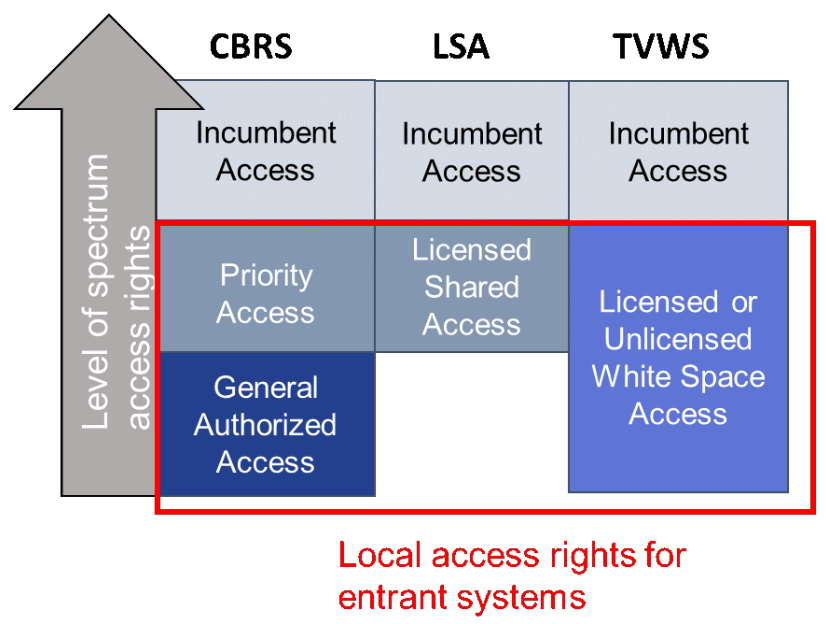

Fig. 2. Existing spectrum sharing models.

includes users under general authorized access (GAA). All tiers have interference protection from lower tiers, and may suffer interference from upper tiers. Spectrum access system (SAS) coordinates and assigns spectrum access rights to the users including databases to keep track and share information of spectrum usage. CBRS is complex sharing model that accommodates different types of spectrum users with the goal of efficient spectrum utilization [7].

European Licensed Shared Access (LSA) is a spectrum sharing model that introduces additional licensed users with guaranteed operational certainty for both incumbents and the entrants [7], [8]. The idea is to allow additional licensed users to access the band under some predetermined rules and conditions while protecting the IUs. Sharing can be done in frequency, time and geographical dimensions. Geolocation databases can be used for spectrum usage decisions. This model has been trial led with a live LTE network in [8]. TVWS spectrum sharing model [9] utilizes unused TV broadcast spectrum in space and time without causing harmful interference to IUs. Typically, geolocation databases are used to ensure interference-free operation for IUs. Database includes, for example, information about TV network infrastructure and channel occupancy, and instruct the PUs about which channels 


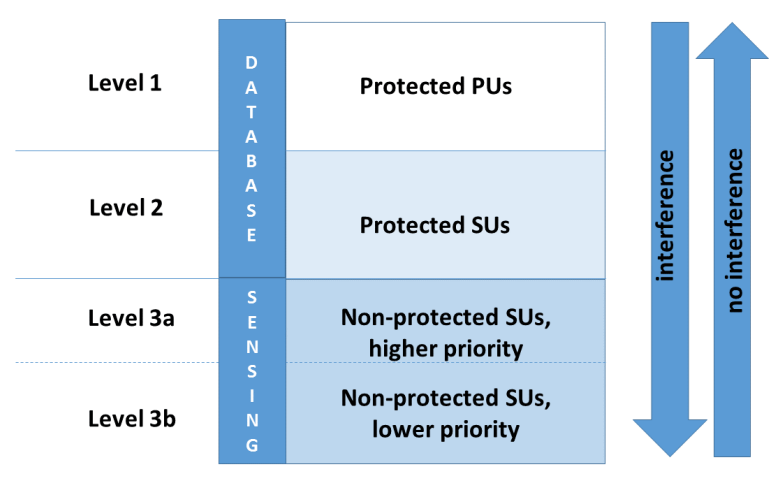

Fig. 3. Proposed spectrum sharing model.

they can use. Spectrum sharing approaches were considered in [10]. Therein it was noticed that because regulatory approaches and IUs differ between countries, national implementations are required.

\section{Proposed Operator ANd Spectrum Sharing MODEL}

Rural and remote areas are potential for new local operator models as discussed in [1], [11]. Localized rural and remote area operators could emerge in spatially confined areas by establishing their own networks provided that there is spectrum available. Since wireless usage in general is lower in rural and remote areas, spectrum could be made available there through shared access. The local rural and remote area operator could serve its own customers directly or it could offer connectivity to MNOs' customers in that area through an agreement with the MNO. The emergence of local rural and remote area operators calls for local spectrum availability. In the proposed spectrum sharing model for remote and rural areas, existing signals are divided into three levels, as illustrated in Figure 3, by following the specification done in [12].

Level 1 consists of incumbent protected PUs specific to the band such as TV signals and wireless microphone signals. Level 2 consists of protected, permanent SU signals that are from built systems meant to operate several months several years. Those include e.g. real-time monitoring systems which have temporary channel allocation. Level 3 consists of unprotected, temporary SU signals. This level can be further divided into two levels: Level 3a includes high priority SU signals like health-related applications like video conference, online doctor, remote monitoring. Level $3 \mathrm{~b}$ includes lower priority SU signals. Level 1 and level 2 signal information are stored in a database. Note, that lower level signals are not allowed to interfere upper level signals.

In rural and remote areas, spectrum sensing is needed to complement databases [13]. Those situations include, for example, when a connection to the database is lost like in crisis situations and under an cognitive attack (hostile/selfish users); transmission powers are small and the transmitter and the receiver are close to each other like in local area network in
TABLE I

SUMMARY OF PROPOSED SPECTRUM SHARING MODEL.

\begin{tabular}{l|l|l|l|l}
$\begin{array}{l}\text { User } \\
\text { type }\end{array}$ & Signal type & $\begin{array}{l}\text { Primary } \\
\text { spectrum } \\
\text { decision } \\
\text { policy }\end{array}$ & $\begin{array}{l}\text { Secondary } \\
\text { spectrum } \\
\text { decision } \\
\text { policy }\end{array}$ & Comment \\
\hline $\begin{array}{l}\text { Level 1 } \\
\text { (PU) }\end{array}$ & $\begin{array}{l}\text { TV signals } \\
\text { (ATSC, NTSC) }\end{array}$ & $\begin{array}{l}\text { Database and } \\
\text { sensing (opt.) }\end{array}$ & $\begin{array}{l}\text { Only } \\
\text { sensing }\end{array}$ & $\begin{array}{l}\text { Fixed } \\
\text { channel } \\
\text { allocation } \\
\text { for PUs }\end{array}$ \\
\hline $\begin{array}{l}\text { Level 2 } \\
\text { (SU) }\end{array}$ & $\begin{array}{l}\text { SUs e.g. } \\
\text { wireless } \\
\text { microphones }\end{array}$ & $\begin{array}{l}\text { Database and } \\
\text { sensing }\end{array}$ & $\begin{array}{l}\text { Only } \\
\text { sensing }\end{array}$ & $\begin{array}{l}\text { Temporary } \\
\text { channel } \\
\text { allocation } \\
\text { for SUs }\end{array}$ \\
\hline $\begin{array}{l}\text { Level 3a } \\
\text { (SU) }\end{array}$ & $\begin{array}{l}\text { high } \\
\text { priority } \\
\text { SUs }\end{array}$ & $\begin{array}{l}\text { Sensing and } \\
\text { database }\end{array}$ & $\begin{array}{l}\text { Only } \\
\text { sensing }\end{array}$ & $\begin{array}{l}\text { No } \\
\text { Permanent } \\
\text { allocation }\end{array}$ \\
\hline $\begin{array}{l}\text { Level 3b } \\
\text { (SU) }\end{array}$ & $\begin{array}{l}\text { low } \\
\text { priority }\end{array}$ & $\begin{array}{l}\text { Sensing and } \\
\text { database }\end{array}$ & $\begin{array}{l}\text { Only } \\
\text { sensing }\end{array}$ & $\begin{array}{l}\text { No } \\
\text { Permanent } \\
\text { allocation }\end{array}$ \\
\hline
\end{tabular}

home; the amount of wireless traffic is small and short term like in case of wireless sensor and other Internet of Things (IoT) devices; transmission powers are small and transmission based on sensing requires a lot less power than asking for a permission; and in local networks in a remote area with only few members like in national parks.

In rural and remote area use cases, spectrum sensing is required at level 3 to find out free channel resources for operation and to enable coexistence of different SU signals in that level. Basically, if upper levels have a connection to the database, the spectrum sharing can be managed using purely that information among devices at those levels. However, when the connection to the database is lost, sensing is required also for level 2 signals to avoid interfering level 1 signals. In addition, we propose that spectrum sensing can be used in level 2 also to improve reliability of decisions, i.e., spectrum sensing capability can be used to guarantee that a frequency band assigned as vacant by the data base is indeed empty, and vice versa. For example, non-authorized broadcasting might be present, or the propagation mechanisms in a given region allow for a DTV receiver to get a signal in an area where should not be coverage based on the propagation prediction software calculation.

Table I summarizes the characteristic of proposed spectrum sharing model. Level 1 includes TV signals that have a fixed channel allocation but sensing can be used optionally to improve reliability. Channel allocations are stored in database which can be used by each layer devices. Level 2 includes SU devices which have a temporary channel allocation, e.g., for few months or years duration. This level includes e.g. wireless microphone signals. Level 2 channel allocations are stored in database. Depending on the situation and scenario, level 2 devices may also use spectrum sensing to avoid interfering of protected PUs at level 1. Level 3 includes SUs that will use the free spectrum available at the TV bands. Devices will use spectrum sensing and channel allocation information available 


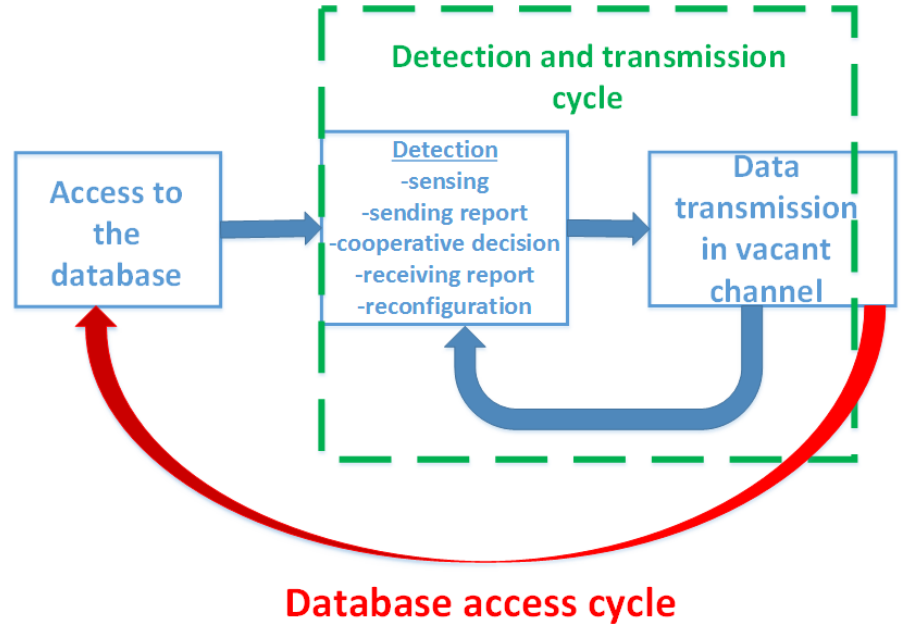

Fig. 4. Database and sensing cycles.

from database to find out free channel opportunities. Spectrum sensing can be used to improve the reliability of decisions and make the free channel resource discovery more dynamic and efficient in comparison to pure database approach. If level 3 devices do not have access to database, spectrum decision will be made based purely on sensing information. Level 3 devices must avoid interfering of upper level users which have fixed or temporary channel allocation. Spectrum sensing (in-band) is needed to notice the appearance of level 1 and level 2 users at the frequency band used by devices. In addition, spectrum sensing will be used to improve coexistence with other users.

\section{Database and Spectrum Sensing}

In the proposed spectrum sharing model, both geolocation database and spectrum sensing are used to find spectrum access opportunites. This process includes database access as well as detection and transmission cycles, as illustrated in Figure 4 following the definition from [14]. Detection includes sensing, sending a report to fusion centre, receiving a cooperative decision, and reconfiguration. In the detection and transmission cycle, detection and data transmission alternate. Database access cycle includes access to the database, detection, and data transmission, and it is performed regularly (typically with spaces of hours).

The database protects the IUs from harmful interference caused by the additional users. The database includes information about authorised transmissions in the given band such as TV signals excluding pirate TV broadcasting, PMSE signals, and information about the wireless systems, such as location, bandwidth, maximum allowed power, and the period of usage. An essential part of the database is geographical information, for example, obstacles like buildings and terrain information including mountains to model the propagation characteristics for the interference studies.

The system includes spectrum sensing in addition to the database approach to find signals that are not listed in the

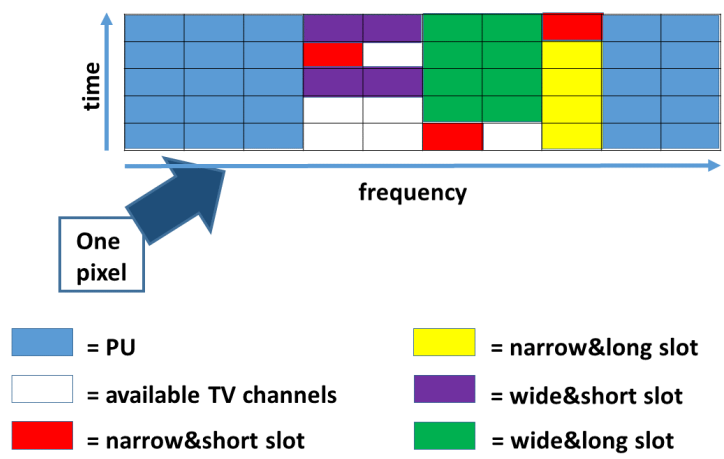

Fig. 5. Time and frequency resource allocation in one geographical pixel. Available channels can be narrow in frequency and short in time, narrow in frequency and long in time, wide in frequency and short in time, and wide in frequency and long in time.

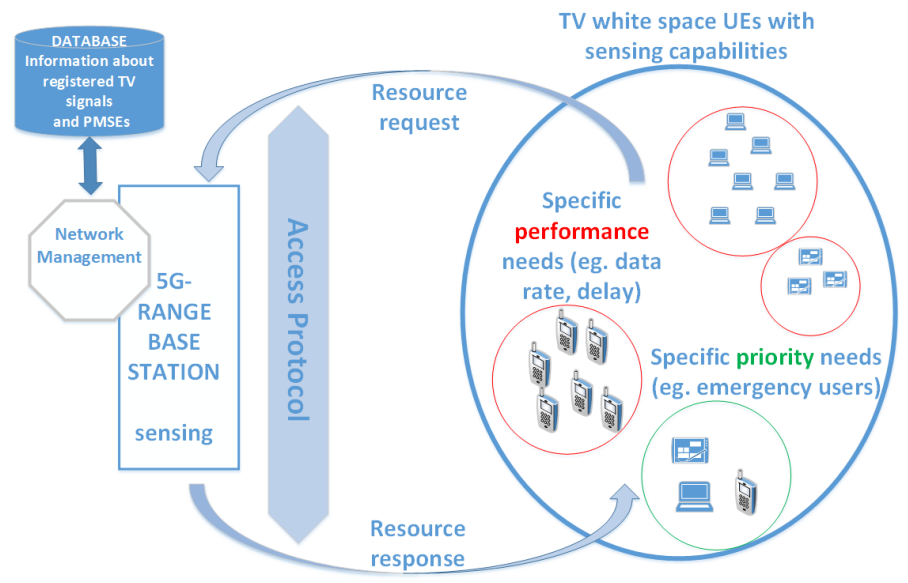

Fig. 6. Different users may have different spectrum and priority needs. For example, video transmission requires more resources than just a voice, and emergency users may have higher priority than other users.

database which adds the complexity of the system but improves the performance. Before sensing, UE provides its location information, transmit coverage area and device type to the base station. This ensures that the base station can allocate resources that are sized to the devices capabilities (bandwidth, maximum transmit power) as illustrated in Figure 5. This allocation uses database information obtained through the network management system. For example, UE downloading some video content gets more resources than UE transmitting just voice. In an emergency case, UEs transmitting critical data may have higher priority than other UEs (Figure 6).

The database sends a list of possible vacant frequencies (channels that are not reserved for registered TV or PMSE devices), and the maximum allowed transmit power values to the base station, which sends the information to the UE. Both time and spatial aspects are taken into account: the channel has to be vacant at that time and on that geographical location. Spectrum occupancy is detected when there is a signal present, so the channel is defined to be occupied. 


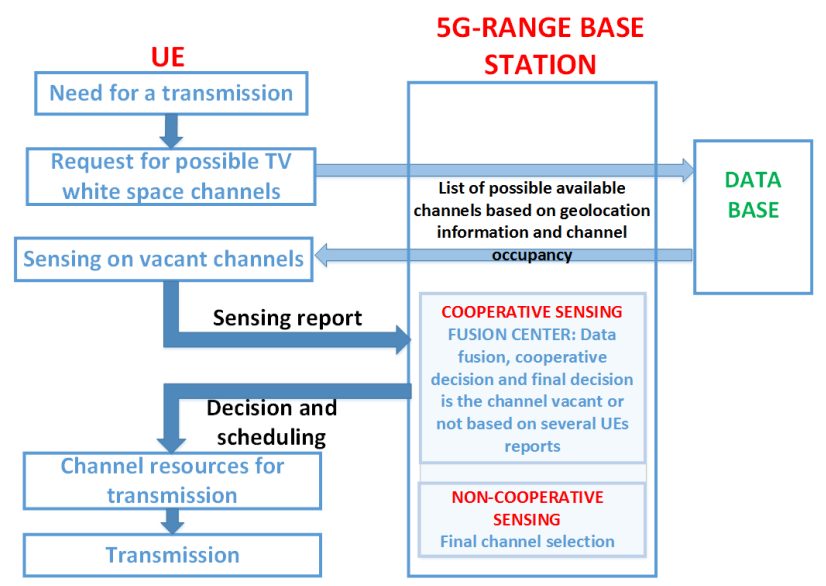

Fig. 7. Combining geolocation database and sensing information.

If the UE can not detect PU signal and starts to transmit, the PU can experience interference. Obstacles like buildings of mountains, shadowing, path loss and fading cause problems to detect PU signals. One possible answer is to use cooperative sensing [15] where sensing results of several UEs are combined. It utilises spatial diversity when sensing devices are in different positions. Centralized cooperation means that a fusion centre controls the sensing and makes the final decision.

The overall process is illustrated in Figure 7. It starts when the UE needs to transmit and sends a resource request to the base station. Base station acquires a list of possible channel candidates that are vacant from PUs based on UEs location information from the database. After that, the gNB may ask UE(s) to perform spectrum sensing to detect if there are unregistered PMSEs, pirate TV channels and other existing (narrowband) signals in the candidate channel(s). In sensing, a specific detection threshold is used when defining if the channel is vacant or occupied. In the case of individual sensing, UE decides if the channel is vacant or not and sends that information to gNB. During the long transmission, inband sensing is required to avoid collisions with other users like PUs. In the case of cooperative sensing, UE sends sensing report to a fusion centre that makes a final decision if the channel is vacant or occupied based on several UEs reports. Fusion centre sends its report to gNB which can then allocate channel resources to UEs.

In some situations, there may be no connection available to the database. This may occur in very sparsely populated rural areas, where, e.g., there is only a single farm (smart farm) or a nature park, or in unexpected disasters, like an earthquake. In that case, UE should try to connect to the database using, for example, an Internet connection, if the database is publicly available. Alternatively, the system can ask the database information for any other nearby UE that may have access to it. If it is impossible to get a connection to the database, pure sensing may be acceptable if some conditions are fulfilled. Those conditions may concern, for example, used channels or used transmit powers. It may also be possible that in areas where it is known that there may be no connection to the database, some specific channels are reserved to UEs where they can transmit using some maximum transmission power based on pure sensing. Those channels should be selected so that there is no PUs nearby. Those channels can also be used in emergency situations. [16-18]

\section{CONCLUSION}

This paper has outlined a spectrum sharing model that allows the establishment of local $5 \mathrm{G}$ networks to serve rural and remote areas which are not covered by the MNO networks. The emergence of local $5 \mathrm{G}$ operators is crucially dependent on the availability of local spectrum which can be achieved through shared access to the spectrum. This paper has presented a general spectrum sharing model that promotes the establishment of local $5 \mathrm{G}$ networks by different stakeholders through a combined database and spectrum sensing approach.

\section{REFERENCES}

[1] J. Saldana et al., "Alternative networks: Toward global access to the internet for all," IEEE Commun. Mag, vol. 55, no. 9, 2017, pp. 187193.

[2] F. Paisana, N. Marchetti, and L. A. DaSilva, "Radar, TV and cellular bands: Which spectrum access techniques for which bands?" IEEE Communications Surveys \& Tutorials.

[3] O. Holland, B. Bochow, and K. Katzis, "IEEE 1900.6b: sensing support for spectrum database," in IEEE Conf. on Standards for Communications and Networking (CSCN), 2015, pp. 199-205.

[4] A. Saeed, M. Ibrahim, K. A. Harras, and M. Youssef, "Toward dynamic real-time geo-location databases for TV white spaces," IEEE Network, vol. 29, no. 5, 2015, pp. 76-82.

[5] 5G-RANGE, "D2.1 - Application and requirements report," 5G-RANGE Project, Tech. Rep., 2018.

[6] FCC, "3.5 GHz band/citizens broadband radio service," Tech. Rep., 2015.

[7] M. Mustonen, M. Matinmikko, D. Robertson, and S. Yrjölä, "Evaluation of recent spectrum sharing models from the regulatory point of view," in Int. conf. on 5G for Ubiquitous Connectivity, 2014.

[8] M. Matinmikko, H. Okkonen, M. Palola, S. Yrjölä, P. Ahokangas, and M. Mustonen, "Spectrum sharing usong licenced shared access: the concept and its workflow for LTE-advanced networks," IEEE Wirel. Commun., 2014.

[9] S. Yrjölä, M. Matinmikko, and M. Mustonen, "Analysis of sharing economy antecedents for recent spectrum sharing consepts," in COCORA, 2017.

[10] M. Matinmikko, M. Mustonen, D. Robertson, J. Paavola, M. Höyhtyä, and S. Yrjölä, "Overview and comparison of recent spectrum sharing approaches in regulation and research - from opportunistic unlicensed access towards licensed shared access," in DYSPAN, 2014.

[11] M. Matinmikko, M. Latva-aho, P. Ahokangas, and V. Seppänen, "On regulations for 5G: Micro licensing for locally operated networks," Telecommunications Policy, vol. 42, no. 8, 2018, pp. 622-635.

[12] 5G-RANGE, "D4.2 - Spectrum Sensing to Complement Databases," 5GRANGE Project, Tech. Rep., 2018.

[13] J. Vartiainen, M. Höyhtyä, and R. Vuohtoniemi, "The future of spectrum sensing," in ICUFN, 2016

[14] 5G-RANGE, "D2.2 - Architecture, system and interface definitions of a 5G for Remote Area network," 5G-RANGE Project, Tech. Rep., 2019.

[15] I. F. Akyildiz, B. F. Lo, and R. Balakrishnan, "Cooperative spectrum sensing in cognitive radio networks: A survey," Physical Communication, vol. 4, no. 1, 2011, pp. 40-62.

[16] D. Lavaux et al., "Final architcture for TVWS spectrum sharing systems," in Cogeu, 2011.

[17] J. C. Ribeiro, "Testbed for combination of local sensing with geolocation database in real environments," IEEE Wirel. Commun., vol. 19, 2012, pp. 59-66.

[18] V. Chen, S. Das, L. Zhu, J. Malayar, and P. McCann, "Protocol to access white-space (PAWS) database," Tech. Rep., 2015. 